\title{
Precise Localization of Iris outer boundary based on the delineation of region of interest
}

\author{
Yang Xiaosheng ${ }^{1, a^{*}}$, Chen Houjin ${ }^{1, b}$, Li Jupeng ${ }^{1, c}$ \\ ${ }^{1}$ School of Electronic \& Information Engineering, Beijing Jiaotong University, Beijing, China \\ a13120040@bjtu.edu.cn, bhjchen@bjtu.edu.cn, cjpli1@bjtu.edu.cn
}

KeyWords: Iris Localization, Region of interest, Edge detection, Hough transform

\begin{abstract}
The prerequisite of a successful iris recognition system is the fast and accurate iris localization. Delineation of the iris outer boundary is more complex than delineation of iris inner boundary, so a precise localization method of iris outer boundary based on the delineation of region of interest was presented in this paper. The result shows that this method could detect the iris outer boundary fast and precisely compared to the traditional methods.
\end{abstract}

\section{Introduction}

Precise localization of the iris boundary is the prerequisite of effective iris recognition and the localization of iris outer boundary needs to be much more concentrated on due to its complexity. At present, there are three main methods to locate the iris outer boundary: 1) Circle detecting based on the differential operator; 2) Edge detecting combined with Hough transform ${ }^{[2]}$; 3) Curve fitting using least square method ${ }^{[3]}$. The first model has high accuracy and strong rubustness while the computation of this method is too heavy due to blind searching ${ }^{[4]}$. The operation time of the second method is too long as well. The third method has reduced the operation time obviously. But its performance could be easily influenced by the threshold ${ }^{[5]}$.

In consideration of the limitations of above three kinds of methods, this paper proposes a precise localization method of Iris outer boundary based on the delineation of region of interest. First of all, we use a rectangular template to detect points on the iris outer boundary along though the horizontal direction which passes the center of iris inner boundary. After that, the region of interest could be delineated on the basis of these points. Finally, in the region of interest, the iris outer boundary could be located precisely and quickly by using edge detecting method combined with Hough transform.

\section{Iris outer boundary localization algorithm}

The iris images used in the process of algorithm research are from CISIA $1.0^{[7]}$ iris library (contains 1307 images) of Chinese academy of sciences. All the images are grayscale images and in the size of 320x280. To improve the pertinence of this paper, we assume the center of the iris inner boundary is $\mathrm{O}\left(\mathrm{x}_{0}, \mathrm{y}_{0}\right)$ and its radius is $\mathrm{R}_{\mathrm{in}}$. The overall flow chart of iris outer boundary localization is shown in figure 1 :

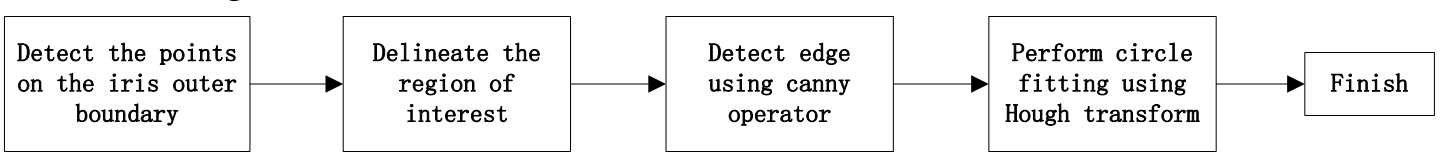

Figure 1 Flow chart of iris outer boundary localization 


\subsection{Select the rectangular template}

The structure of the human eye is shown in figure2. The gray value changes obviously at the junction between iris and sclera. The change reflects in the difference between the grayscale sums of two adjacent point sets which are separated by the point on the iris outer boundary. On account of this feature, we have designed a rectangular template to detect the iris outer boundary. The template is shown in figure $3(\mathrm{~W}=\mathrm{H}=5$ pixels). Because there is hardly any eyelash and some other noise in the horizontal direction which passes the center of iris inner boundary, we use the rectangular template to detect the iris outer boundary in this direction.

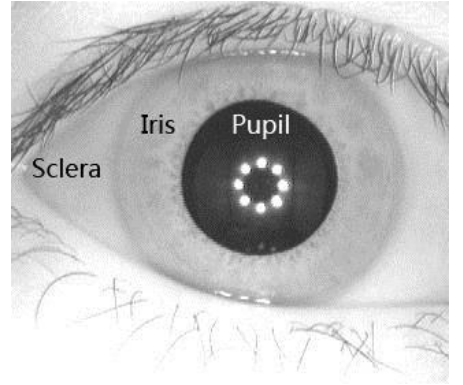

Figure 2 The structure of human eye

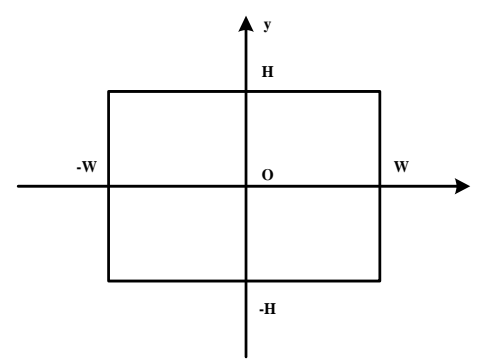

Figure 3 Rectangular Template

\subsection{Limit the rang of detection}

In order to reduce computation and improve the accuracy of delineation, we need to determine the range of iris outer boundary detection flexibly based the radius and center of iris inner boundary. The detection of iris left outer boundary is the same with the detection of its right outer boundary, so we only detect the left outer boundary in this paper. The result is very satisfactory when we use the equation (1 ) (2) to determine the detection range, which is verified by a number of experiments. $\mathrm{P}_{\text {lmin }}$ and $\mathrm{P}_{\operatorname{lmax}}$ are the start point and end point of the range of iris left outer boundary detection.

$$
\begin{gathered}
P_{l \min }= \begin{cases}{\left[O_{i n}-3.7 * R_{i n}\right]} & ,\left(R_{i n}<33\right) \\
{\left[O_{i n}-3 * R_{i n}\right]} & ,\left(33 \leq R_{i n}<38\right) \\
{\left[O_{i n}-2.5 * R_{i n}\right]} & ,\left(38 \leq R_{i n}<45\right) \\
{\left[O_{i n}-2.1 * R_{i n}\right]} & ,\left(R_{i n} \geq 45\right)\end{cases} \\
P_{l \max }= \begin{cases}{\left[O_{i n}-3 * R_{i n}\right]} & ,\left(R_{i n}<33\right) \\
{\left[O_{i n}-2 * R_{i n}\right]} & ,\left(33 \leq R_{i n}<38\right) \\
{\left[O_{i n}-1.7 * R_{i n}\right]} & ,\left(38 \leq R_{i n}<45\right) \\
{\left[O_{i n}-1.5 * R_{i n}\right]} & ,\left(R_{i n} \geq 45\right)\end{cases}
\end{gathered}
$$

\subsection{Delineate the ROI(region of interest)}

With the purpose of detecting the iris left outer boundary, the process of detection with the rectangular template starts from the point determined by equation (1) and end with the point determined by equation (2). We calculate the difference of the grayscale sums of the left and right part of the template. The calculation process is shown by the following formula. 


$$
L\left(x_{0}, y\right)=\left|\sum_{i=1}^{W} \sum_{j=-H}^{H} I\left(\left(x_{0}-i\right),(y-j)\right)-\sum_{i=1}^{W} \sum_{j=-H}^{H} I\left(\left(x_{0}+i\right),(y+j)\right)\right|
$$

In equation (3), I represents the iris image. Using this equation, we can find out the point $\mathrm{A}\left(\mathrm{x}_{1}\right.$, $\left.\mathrm{y}_{1}\right)$ where $\mathrm{L}\left(\mathrm{x}_{0}, \mathrm{y}\right)$ reach its maximum value. $\mathrm{A}$ is the point on the left outer boundary and the distance between $A$ and $O$ is represented by $D_{1}$. Likewise, we can find point $B\left(x_{2}, y_{2}\right)$ on the right outer boundary and $\mathrm{D}_{\mathrm{r}}$ (the distance between $\mathrm{B}$ and $\mathrm{O}$ ).

The distance between the center of iris outer boundary and the center of its inner boundary is relatively short. Therefore, $D_{1}$ and $D_{r}$ are not the radius of the outer boundary, but we can obtain the range of the radius on basis of them ${ }^{[6]}$. The range, $R_{\text {annulus, }}$ is determined by the formula (4). In conclusion, we can delineate the ROI, an annulus, whose center is $\mathrm{O}$ and radius range is $\mathrm{R}_{\text {annulus. }}$ Figure 4 shows the delineation of ROI.

$$
\text { mik } D l-8, \quad D r-8) \leq R_{\text {annulus }} \leq \max (D l+8, \quad D r+8)
$$

\subsection{Delineate the iris outer boundary}

In the region of interest, canny operator is used to detect edge. Then, we get a series of points on the boundary. Finally, the outer boundary can be delineated by using Hough transform to perform circle fitting with these points. Figure 5 shows the image after filtering by canny operator and Figure 6 show the localization result.

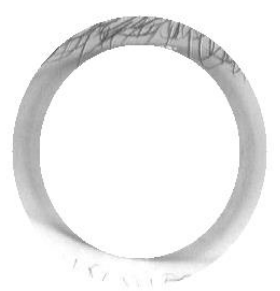

Figure 4 Region of interest

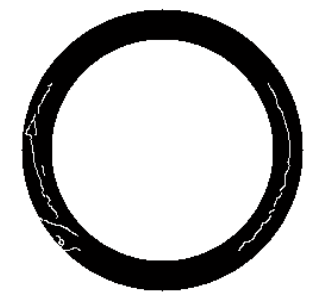

Figure 5 Filtered image

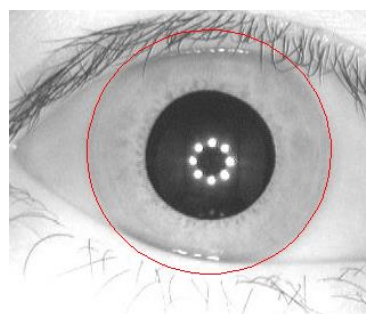

Figure 6 Localization result

\section{Experimental results and analysis}

The localization algorithm in this paper is practiced and simulated under the environment of Matlab 7. 0 .Table 1 shows the performance of the algorithm in this paper and the other existing algorithms.

Table 1 Localization of iris outer boundary

\begin{tabular}{ccc}
\hline Algorithm & Average time[s] & Accuracy[\%] \\
\hline Literature[1] & 5.65 & 95.38 \\
Literature[2] & 4.87 & 93.56 \\
Literature[3] & 1.52 & 96.33 \\
This paper & 0.98 & 98.67 \\
\hline
\end{tabular}

Table 1 shows that we can localize the iris outer boundary precisely and quickly by the algorithm presented in this paper. On one hand, the algorithm used to delineate the ROI has small amount of computation. On the other hand, the delineated ROI is as small as possible, so that the 
amount of points taking part in the following complex computation can be decreased. Meanwhile, precisely delineation of ROI can effectively eliminate the influence of some noise.

\section{Summary}

In this paper, a precise localization method of iris outer boundary based on the delineation of region of interest is presented. By delineate the ROI with an effective method, which has a small amount of calculation, the iris outer boundary can be localized more precisely and more quickly than the existing methods. In conclusion, this algorithm is really helpful for the iris recognition.

\section{Acknowledgement}

This paper is supported by Beijing Municipal Science and Technology Project (D15110000041 5002), Specialized Research Fund for the Doctoral Program of Higher Education (20120009120001 ), and Fundamental Research Funds for the Central Universities (2015JBM015).

\section{References}

[1] Daugman J. How iris recognition works [J]. IEEE Transactions on Circuit and System for Video Technology, 2004, 14(1):21-30.

[2] Wildes R. Iris recognition: an emerging biometric technology [J]. Proceedings of the IEEE, 1997, 85(9): 1348-1363.

[3] Wang Yunhong. Biometrics personal odentification base on iris pattern [J]. Acta Automatica Sinica, 2002, 28(1): 1-10.

[4] Liang Yan, Zeng Biqing. Iris location method based on region growing and Hough transform [J]. Computer Engineering and Application, 2012, 48(8): 200-202.

[5]Yuan Weiqi, Lin Zhonghua. Novel iris location algorithm based on the structure of human eyes [J]. Opto-Electronic Engineering, 2007, 34(1): 112-116.

[6]Ma Zheng, Huang Yi, Improved iris location algorithm based on curve fitting [J]. Journal of University of Electronic Science and Technology of China, 2009, 38(3): 426-430.

[7] CASIA Iris Image Database V-1.0, http://www.cbsr.ia.ac.cn/IrisDatabase.htm, 2013. 